\title{
Clinical Significance of Long Non-Coding RNA HCG18, OIP5-AS1, FGD5-AS1 and LINC00657 Expression in Human Gastric Cancer
}

\section{Ali Zareh}

Baqiyatallah University of Medical Sciences

\section{Elmira Rabani}

Islamic Azad University of Tehran: Islamic Azad University Central Tehran Branch

Hamed Zare

Kerman University of Medical Sciences

\section{Mohammad Heiat}

Baqiyatallah University of Medical Sciences

Hamid Bakherad ( $\nabla$ bakheradh@pharm.mui.ac.ir)

Isfahan University of Medical Sciences https://orcid.org/0000-0002-6740-4267

\section{Primary research}

Keywords: Gastric cancer, IncRNA expression, qPCR, Bioinformatics

Posted Date: November 22nd, 2021

DOI: https://doi.org/10.21203/rs.3.rs-1067282/v1

License: (9) This work is licensed under a Creative Commons Attribution 4.0 International License. Read Full License 


\section{Abstract \\ Background}

Gastric cancer (GC) is a major health problem and the third cause of cancer-induced deaths globally. Long non-coding RNAs (IncRNAs) are new cellular regulators in cancers whose contribution to GC carcinogenesis is still unknown to a large extent. This study aimed to investigate expression levels of bioinformatically ranked IncRNAs in GC tissues.

\section{Methods}

Using a bioinformatic approach, IncRNAs supposed to be involved in GC tumorigenesis were prioritized. Further, the top-ranked IncRNAs including HCG18, OIP5-AS1, FGD5-AS1, and LINC00657 were selected for experimental validation. Moreover, qPCR was used to validate bioinformatics findings in $35 \mathrm{GC}$ and 35 paired adjacent normal gastric tissue samples (ANGTs). Receiver operating characteristic (ROC) curves and area under the ROC curve (AUC) were generated to assess the diagnostic values of the IncRNAs.

\section{Results}

IncRNA-HCG18 showed to be the top-ranked IncRNA involved in GC, as identified by bioinformatics analysis. Additionally, HCG18, OIP5-AS1, FGD5-AS1, and LINC00657 expression levels significantly increased in GC tissue samples compared to ANGTs. The area under the curve (AUC) of HCG18, OIP5-AS1, FGD5-AS1, and LINC00657 were $0.80,0.74,0.73$, and 0.71 , respectively. Considering the findings, it was concluded that HCG18, OIP5-AS1, FGD5-AS1, and LINC00657 IncRNAs may contribute to GC tumorigenesis.

\section{Conclusion}

This study also found that the bioinformatic approach could be applied as an efficient approach to finding candidate IncRNAs relevant to GC progression.

\section{Introduction}

Gastric cancer (GC) is regarded as the third oncological cause of death worldwide. Although great efforts have been made to reduce the GC progression, the mortality rate of GC is still high [1]. Nowadays, GC is a major clinical challenge because this is usually asymptomatic in early-stage and diagnosed at late-stage characterized by a poor prognosis as well as limited treatment options [2]. Accordingly, detailed mechanisms of GC tumorigenesis are still unclear. Hereupon, comprehending how these mechanisms work might contribute to the formation of novel diagnostic and prognostic biomarkers [3]. Long noncoding RNAs (IncRNAs) are greater than 200 nucleotides in length and possess no protein-coding 
potential. They have been identified as novel gene regularizers acting via multiplex methods, including transcriptional modulation, microRNA competition, histone modification, and chromatin remodeling [4]. The present documents suggested that IncRNAs are involved in the control of diverse biological procedures, including metabolism, differentiation, cellular senescence, apoptosis, survival, and tumorigenesis [5, 6]. MicroRNAs (miRNAs) are also the regulators of gene expression which are a family of endogenous non-coding short RNA molecules [7]. It is acknowledged that numerous IncRNAs serve as competing endogenous RNAs (ceRNAs) and sponging specified microRNAs [4]. This kind of IncRNAs competes with miRNAs for binding to the target and lessening miRNA-mediated inhibitory effects on gene expression. Therefore, the IncRNAs create a regulatory axis to control the gene expression [4]. Figure 1 illustrates the roles of HCG18, OIP5-AS1, FGD5-AS1, and LINC00657 in the tumorigenesis process to show how these IncRNAs influence different steps in this process [8-12]. In literature related to GC, manifold IncRNAs are known to be aberrantly expressed, and in their abnormal expression, they remarkably act on the malignant characteristics of GC by altering miRNA expression [8, 13-15]. In considering such findings, it is likely to assume that IncRNAs can regulate tumor progression; hence, the investigation of specific roles of GC-related IncRNAs may propose an efficient target for anticancer therapy. Therefore, this study strived to (I) prioritize IncRNAs involved in GC by bioinformatic resources and (II) investigate the expression levels of candidate IncRNAs in clinical samples.

\section{Materials And Methods}

\section{In-silico analysis}

A general schema of the bioinformatic pipeline is depicted in Figure 2 [16]. LncRNAs can target miRNAs and then indirectly affect mRNAs, so it is possible that the dysregulation of IncRNAs that have further target miRNAs indirectly affects more mRNAs and more cellular/molecular pathways than IncRNAs with fewer miRNAs targets. Therefore, a bioinformatic method was designed to centralize the study on IncRNAs with more target genes as fundamental regulators in GC.

At first, the expression profiles of GC differentially expressed (DE) IncRNAs and miRNAs were downloaded from the Cancer RNA-Seq Nexus (http://syslab4.nchu.edu.tw) [17] and dbDEMC 2.0 online web-servers (https://www.picb.ac.cn/dbDEMC/) [18], respectively. Indeed, the expression profiles of DE-IncRNAs in the Cancer Genome Atlas-Stomach Adenocarcinoma (TCGA-STAD) dataset downloaded from the Cancer RNA-Seq Nexus web-server were explored [17]. This dataset was generated through RNA-seq experiments and included 431 transcriptome profiles comprising 35 noncancerous gastric tissues and 396 gastric tumor samples. Using a tool implemented in the Cancer RNA-Seq Nexus web-server[17], the TCGA-STAD dataset was analyzed to identify DE genes in GC (GC-DE IncRNAs).

At the next step, two aforementioned datasets were cross-referenced with a bulk dataset including comprehensive interactions of IncRNA-miRNA obtained from starBase 2.0 (http://starbase.sysu.edu.cn) [19] and "IncRNA-miRNA implicated in GC" dataset created. Finally, IncRNAs were listed in a created 
dataset, ranked according to the numbers of target miRNAs, and top-ranked outputs were considered as "IncRNAs-implicated in GC."

\section{Patient samples}

The expression levels of IncRNAs were determined in 70 gastric tissue samples including gastric tumors $(n=35,20$ diffuse subtypes and 15 intestinal subtypes) and paired adjacent normal gastric tissues (ANGTs) ( $\mathrm{n}=35$, histologically normal samples with at least three $\mathrm{cm}$ distance from tumor).

Tissues were attained during surgery prior to receiving chemo/radiotherapy. Then, samples were immediately frozen and transferred to the laboratory for later assessments. Samples were stored at $-70^{\circ} \mathrm{C}$ until expression analysis. All specimens were examined by a pathologist to prove the pathologic signs. The study protocol was approved by the Ethical Committee (IR.BMSU.REC.1398.281) in Baqiatallah Medical University. All subjects gave written informed consent in accordance with the Declaration of Helsinki.

\section{RNA extraction and quantification of IncRNAs}

Total RNAs from tissue samples were exploited using the AccuZolTM total RNA extraction kit (Bioneer, Korea) according to the manufacturer's instructions. cDNA from RNA was synthesized by the PrimeScript RT reagent kit (Takara Bio, Ohtsu, Japan). The qPCRs were conducted with SYBR Green Premix Ex Taq (TaKaRa, Otsu, Shiga, Japan) and according to the standard program on Rotor-Gene Q instrument (QIAGEN, Germany). The GAPDH gene was used for the normalization of expression data. Moreover, a non-template control reaction was used for every primer set to certify reaction specificity. The primer sequences have been presented in Table 1.

\section{Statistical analysis}

The quantitative variables were presented as mean \pm standard error of the mean (SEM). Statistical analyses were performed using Graph-pad Prism 7 (RRID: SCR_002798). The IncRNA expression data were examined for normality with the Shapiro-Wilk test. The paired t-test was used to compare the IncRNA expression between two defined groups. ROC curve analysis (ROC) was then performed to measure the diagnostic value of IncRNAs, using MedCalc Version 18.5 (RRID: SCR_015044). A P-value $\leq$ 0.05 was considered to be statistically significant.

\section{Results}

\section{In-silico results}

The IncRNA-miRNA pairs in which target miRNAs showed differential expression in the GC, were identified. More than 1700 discrete IncRNA-target miRNA pairs were discovered. Thereafter, IncRNAs were prioritized based on the number of their target miRNAs. Table 2 shows the top 10 ranked IncRNAs found to be involved in GC (Supplementary File S1 for a complete list). 


\subsection{Transcript Quantities of IncRNAs}

For experimental assays, the study was limited to investigating those ranked-IncRNAs that have not been vastly explored regarding GC tumorigenesis. Therefore, MALAT1, KCNQ10T1, and IncRNA NEAT1 which had been investigated broadly had to be excluded. Taking into consideration all the above-mentioned limitations, the present study assessed the expression levels of HCG18, OIP5-AS1, FGD5-AS1, and LINC00657. The results revealed that expression levels of HCG18, OIP5-AS1, FGD5-AS1, and LINC00657 are significantly upregulated in the GC group compared with the ANGTs group as determined by paired ttest (Table 3, Figure 3).

\section{Correlation between the four-IncRNA signature and clinicopathological features of gastric cancer patients}

Patients' demographic data are presented in Table 4. The correlation was examined between clinicopathological variables and expression levels of HCG18, OIP5-AS1, FGD5-AS1, and LINC00657 in the GC group. Table 5 shows the summary of the results. Accordingly, no meaningful difference was obsereved between HCG18, OIP5-AS1, FGD5-AS1, and LINC00657 expression levels in the GC group concerning patient's age and sex $(P>0.05)$. The statistical analyses also revealed no significant correlation of studied IncRNAs with $\mathrm{H}$. pylori infection except for OIP5-AS1, which was near-threshold pValue and marginally significant $(P=0.06)$.

In the case of HCG18, results showed a significant upregulation of this gene expression in advancedgrade samples $(P=0.04)$, but its upregulated expression had no notable correlation with tumor size, lymph node metastasis $(L N M)$, or $\mathrm{H}$. pylori infection $(P>0.05)$. The results also revealed that OIP5-AS1 is obviously upregulated in LNM positive specimens $(P=0.01)$. Morover, it was detected that the OIP5-AS1 expression level is upregulated in tumor samples with higher developed grades $(I+I I$ vs. III+IV, $P=0.01)$ as well as tumors with a larger size $(P=0.007)$. Regarding FGD5-AS1, although remarkable upregulation was found in both LNM positive samples $(P=0.008)$ and tumor tissues greater than $5 \mathrm{~cm}$ in size $(P=0.01)$, there was no significant difference regarding cancer grade.

Moreover, results showed that LINC00657 significantly overexpresses in tissue samples with more advanced clinical-grade $(P=0.01)$ and those with positive LNM $(P=0.003)$; however, no significant difference regarding tumor size was observed.

\section{Evaluation of Diagnostic Power of IncRNA}

To determine if the expression of four investigated IncRNAs has the potential to discriminate between healthy and unhealthy subjects, a receiver operating characteristic (ROC) curve was illustrated. Analyses of ROC curve outputs demonstrated that HCG18 has the greatest diagnostic power (area under the curve $(A U C)=0.80$, P-value <0.0001). OIP5-AS1, LINC00657, and FGD5-AS1 are ranked thereafter (AUC values= $0.74,0.73$ and $0.71 ; P$ values $=<0.0001,0.0004$ and 0.0005 , respectively) (Figure 4 ). The detailed evaluation of ROC curve parameters is presented in Table 6. 


\section{Discussion}

In this research, an integrative bioinformatics approach was applied to choose IncRNAs involved in GC. It is commonly agreed that IncRNAs can regulate tumor development by sequestering miRNAs (microRNA), releasing their downstream target mRNAs, and prompting mRNAs to exert functions $[14,15]$. These findings imply that aberrantly expressed IncRNAs exert an important act on the malignant trait of cancer by changing miRNA (microRNA) expression. Taking this into consideration,IncRNAs were arranged according to the number of their target miRNAs. Then, in order to focus the study on gastric cancerrelated non-coding RNAs precisely, at first, candidate IncRNAs were limited to those which are GC differentially expressed based on the Cancer Genome Atlas-Stomach Adenocarcinoma (TCGA-STAD) data. Secondly, the targeted miRNAs were cross-referenced with GC differentially expressed miRNAs in the dbDEMC 2.0 database. As seen in Table 2, HCG18 has been recognized as the top-level IncRNA involved in GC, with 69 different target miRNAs. To further examine the bioinformatics findings, the expression levels of selected IncRNAs, including HCG18, OIP5-AS1, FGD5-AS1, and LINC00657 were analyzed. The obtained results revealed that these four IncRNAs are obviously upregulated in GC specimens. In some recent investigations, various dysregulated oncogenic IncRNAs have been also reported in human cancers $[8,20,21]$.

HCG18 is characterized as a 2430-bp IncRNA on chromosome 6p22.1. Recently, it has been known as a novel cancer-related IncRNA which repeatedly upregulates and acts chiefly as an oncogene in different cancers including colorectal cancer and nasopharyngeal carcinoma $[8,13]$. For example, Li et al. verified that HCG18 is involved in regulating the MTDH gene (a potential oncogene in colorectal cancer) by sponging miR-1271[13]. To date, the implication of HCG18 in GC advancement is still unclear. Results revealed that HCG18 significantly overexpresses in GC samples (ANGT vs. GC, $P=<0.0001$ ). Although these results demonstrated a meaningful increase of HCG18 expression in samples with advanced tumor grade $(P=0.04)$, no significant correlation was detected between HCG18 expression level and LNM positivity or size of the tumor. Likewise, a recent study has revealed that HCG18 is overexpressed in GC tissues, which supports this work results and indicates that HCG18 acts as a tumor promoter in GC [22]. The involvement of HCG18 in determining metastatic features and tumor invasion has already been reported [22]. The above-mentioned contradictions could be described partly by both biological pathway redundancies in tumor biology as well as tumor heterogeneity. Some recent investigations have further shown that IncRNA HCG18 promotes cancers by modulating PI3K/Akt, Wnt/ $\beta$-catenin, and Hedgehog signaling pathways $[8,22]$.

OIP5-AS1 is a novel and promising IncRNA that is located on chromosome 15q15.1. According to the results, OIP5-AS1 is upregulated in GC samples (GC vs. ANGTs, $P=0.0015)$, especially in higher grades, larger tumors, and LNM positive samples which highlights its function in tumor progression. It has been also proposed that over-expression of OIP5-AS1 considerably promotes the tumorigenicity and proliferation of GC cells; however, its clinical importance in GC has remained unknown. Results of the current work agree with those of the previous studies by Bai et al. and Tao et al. They declared that OIP5AS1 is overexpressed in GC tissues and cell lines [21,23]. In a recent investigation, the analysis of cell 
cycle on osteosarcoma revealed that silencing of OIP5-AS1 could give rise to the apoptosis of tumor cells, prevention of the cell cycle at G0/G1 phase, and consequently inhibit tumor growth [9]. Accordingly, various studies suggested the oncogenic role of OIP5-AS1 in multiple human cancers including hepatoblastoma and cervical cancer [24, 25].

In this study,it was demonstrated that the expression level of FGD5-AS1 is remarkably upper in GC specimens than ANGTs. Meantime, the upregulated FGD5-AS1 was meaningfully associated with larger tumor size and LNM positive specimens. Dysregulation of FGD5-AS1 has been also reported in various types of malignancies [10,20,26]. Additionally, it has been discovered that FGD5-AS1 is aberrantly upregulated in invasive colorectal cancer and FGD5-AS1 knockdown significantly weakens cell invasion and migration potential. Moreover, certain evidence has suggested that significant upregulation of FGD5AS1 could promote colorectal cancer invasion and metastasis through upregulating CDCA7 (a cancer cell metastasis mediator) [10].

In addition, the LINC00657 dysregulated expression has already been reported in various tumors [12, 27, 28]. However, the direction of dysregulation has remained conflicting. An investigation on colon cancer indicated that LINC00657 could promote the expression of CAPN7 gene and thus inhibit cell invasion [28]. In contrast, pancreatic ductal adenocarcinoma is characterized by LINC00657 overexpression in tissue specimens [12]. Atypical expression of this IncRNA begins cell transformation toward malignancy and tumorigenesis by activating RhoA/ROCK1 pathway [29]. In the current study, it wasfound that expression of LINC00657 is considerably upregulated in GC samples compared with normal ones. In addition, LNM positive samples and also higher-grade tumors increased LINC00657 expression proposing the correlation between this IncRNA and the risk of developing tumors.

\section{Conclusions}

In this research, we showed that applying a bioinformatic approach could beneficially prioritize IncRNAs involved in GC. In addition, the up-regulation of HCG18, OIP5-AS1, FGD5-AS1, and LINC00657 was found in tumor tissues compared with ANGTs. Furthermore, the ROC curve outputs revealed that four candidate genes could differentiate gastric samples from normal gastric tissue with high accuracy. However, it should be pointed out that the results of this study were based on a relatively limited number of subjects and clinicopathological variables; thus, confirmation is required by analyzing larger cohorts.

\section{Declarations}

Author Contributions: A.Z., HZ, analyzed the data; A.Z., H.Z., data curation; A.Z., H.Z., wrote the manuscript; H.B., E.R. performed the experiment; M.H., H.B., H.Z., writing-review and editing M.H., H.B., H.Z, supervision, M.H. project administration.

\section{Funding:}

None 


\section{Ethics approval and consent to participate}

The study was approved by Baqiatallah University of Medical Sciences Ethics Committee (IR.BMSU.REC.1398.281) and performed in compliance with Helsinki declaration.

\section{Acknowledgments:}

The authors would like to thank the Clinical Research Development Unit of Baqiyatallah Hospital, Tehran, Iran, for guidance and advice.

\section{Competing interests}

The authors declare that they have no fnancial competing interests

\section{References}

1. Bray, F.; Ferlay, J.; Soerjomataram, I.; Siegel, R.L.; Torre, L.A.; Jemal, A. Global cancer statistics 2018: GLOBOCAN estimates of incidence and mortality worldwide for 36 cancers in 185 countries. CA: Cancer. J. Clin. 2018, 68, 394-424. doi:10.3322/caac.21492

2. Hohenberger, P.; Gretschel, S. Gastric cancer. Lancet 2003, 362, 305-315. doi:10.1016/S01406736(03)13975-X

3. Zhou, J.; Huang, H.; Tong, S.; Huo, R. Overexpression of long non-coding RNA cancer susceptibility 2 inhibits cell invasion and angiogenesis in gastric cancer. Mol. Med. Rep. 2017, 16, 5235-5240. doi:10.3892/mmr.2017.7233

4. Zhang, G.; Pian, C.; Chen, Z.; Zhang, J.; Xu, M.; Zhang, L.; Chen, Y. Identification of cancer-related miRNA-IncRNA biomarkers using a basic miRNA-IncRNA network. PLOS ONE 2018, 13, e0196681. doi:10.1371/journal.pone.0196681

5. Zhao, J.; Li, L.; Han, Z.Y.; Wang, Z.X.; Qin, L.X. Long noncoding RNAs, emerging and versatile regulators of tumor-induced angiogenesis. Am. J. Cancer Res. 2019, 9, 1367-1381. PMCID: PMC6682713

6. Jiang, M.C.; Ni, J.J.; Cui, W.Y.; Wang, B.Y.; Zhuo, W. Emerging roles of IncRNA in cancer and therapeutic opportunities. Am. J. Cancer Res. 2019, 9, 1354-1366.

7. Bartel, D.P. MicroRNAs: genomics, biogenesis, mechanism, and function. Cell 2004, 116, 281-297. doi:10.1016/s0092-8674(04)00045-5

8. Li, L.; Ma, T.T.; Ma, Y.H.; Jiang, Y.F. LncRNA HCG18 contributes to nasopharyngeal carcinoma development by modulating miR-140/CCND1 and Hedgehog signaling pathway. Eur. Rev. Med. Pharmacol. Sci. 2019, 23(23), 10387-10399. doi:10.26355/eurrev_201912_19678

9. Dai, J.; Xu, L.; Hu, X.; Han, G.; Jiang, H.; Sun, H.; Zhu, G.; Tang, X. Long noncoding RNA OIP5-AS1 accelerates CDK14 expression to promote osteosarcoma tumorigenesis via targeting miR-223. Biomed. Pharmacother. 2018, 106, 1441-1447. Doi:10.1016/j.biopha.2018.07.109 
10. Li, D.; Jiang, X.; Zhang, X.; Cao, G.; Wang, D., Chen, Z. Long noncoding RNA FGD5-AS1 promotes colorectal cancer cell proliferation, migration, and invasion through upregulating CDCA7 via sponging miR-302e. In Vitro Cell Dev. Biol. Anim. 2019, 55(8), 577-585. doi:10.1007/s11626-01900376-x

11. Li, S.; Huang, J.; Qin, M.; Zhang, J.; Liao, C. High expression of CDCA7 predicts tumor progression and poor prognosis in human colorectal cancer. Mol. Med. Rep. 2020, 22(1), 57-66. doi:10.3892/mmr.2020.11089

12. Bi, S.; Wang, Y.; Feng, H.; Li, Q. Long noncoding RNA LINC00657 enhances the malignancy of pancreatic ductal adenocarcinoma by acting as a competing endogenous RNA on microRNA-433 to increase PAK4 expression. Cell Cycle 2020, 19(7), 801-816. doi:10.1080/15384101.2020.1731645

13. Li, S.; Wu, T.; Zhang, D.; Sun, X.; Zhang, X. The long non-coding RNA HCG18 promotes the growth and invasion of colorectal cancer cells through sponging miR-1271 and upregulating MTDH/Wnt/ $\beta$ catenin. Clin. Exp. Pharmacol. Physiol. 2020, 47, 703-712. doi:10.1111/1440-1681.13230

14. Dong, Y.; Wang, G. Knockdown of IncRNA SNHG12 suppresses cell proliferation, migration and invasion in breast cancer by sponging miR-451a. Int. J. Clin. Exp. Pathol. 2020, 13(3), 393-402.

15. Xu, F.; Zhang, J. Long non-coding RNA HOTAIR functions as miRNA sponge to promote the epithelial to mesenchymal transition in esophageal cancer. Biomed Pharmacother. 2017, 90, 888-896. doi:10.1016/j.biopha.2017.03.103

16. Blankenberg, D.; Johnson, J.E.; Taylor, J.; Nekrutenko, A. Wrangling Galaxy's reference data. Bioinformatics 2014, 30, 1917-1919. doi:10.1093/bioinformatics/btu119

17. Li, J.R.; Sun, C.H.; Li, W.; Chao, R.F.; Huang, C.C.; Zhou, X.J.; Liu, C.C. Cancer RNA-Seq Nexus: a database of phenotype-specific transcriptome profiling in cancer cells. Nucleic Acids Res. 2016, 44, D944-951.doi:10.1093/nar/gkv1282

18. Yang, Z.; Wu, L.; Wang, A.; Tang, W.; Zhao, Y.; Zhao, H.; Teschendorff, A.E. dbDEMC 2.0: updated database of differentially expressed miRNAs in human cancers. Nucleic Acids Res. 2016, 45, D812D818. doi:10.1093/nar/gkw1079

19. Li, J.-H.; Liu, S.; Zhou, H.; Qu, L.-H.; Yang, J.-H. StarBase v2.0: decoding miRNA-ceRNA, miRNA-ncRNA and protein-RNA interaction networks from large-scale CLIP-Seq data. Nucleic Acids Res. 2014, 42, D92-7. doi:10.1093/nar/gkt1248

20. Liu, L.; Zhan, Y.; Huang, Y.; Huang, L. LncRNA FGD5-AS1 can be predicted as therapeutic target in oral cancer. J Oral Pathol Med. 2020, 49(3), 243-252. doi:10.1111/jop.12989

21. Tao, Y.; Wan, X.; Fan, Q.; Wang, Y.; Sun, H.; Ma, L.; Sun, C.; Wu, Y. Long non-coding RNA OIP5-AS1 promotes the growth of gastric cancer through the miR-367-3p/HMGA2 axis. Dig. Liver Dis. 2020, 52(7), 773-779. doi:10.1016/j.dld.2019.11.017

22. Ma, F.; An, K.; Li, Y. Silencing of Long Non-Coding RNA-HCG18 Inhibits the Tumorigenesis of Gastric Cancer Through Blocking PI3K/Akt Pathway. OncoTargets Ther. 2020, 13, 2225-2234. doi:10.2147/OTT.S240965 
23. Bai, Y.; Li, S. Long noncoding RNA OIP5-AS1 aggravates cell proliferation, migration in gastric cancer by epigenetically silencing NLRP6 expression via binding EZH2. J. Cell. Biochem. 2020, 121, 353362. doi:10.1002/jcb.29183

24. Zhang, Z.; Liu, F.; Yang, F.; Liu, Y. Kockdown of OIP5-AS1 expression inhibits proliferation, metastasis and EMT progress in hepatoblastoma cells through up-regulating miR-186a-5p and down-regulating ZEB1. Biomed. Pharmacother. 2018, 101, 14-23. doi:10.1016/j.biopha.2018.02.026

25. Song, L.; Wang, L.; Pan, X.; Yang, C. IncRNA OIP5-AS1 targets ROCK1 to promote cell proliferation and inhibit cell apoptosis through a mechanism involving miR-143-3p in cervical cancer. Braz. J. Med. Biol. Res. 2020, 53, e8883. doi:10.1590/1414-431X20198883

26. Fan, Y.; Li, H.; Yu, Z.; Dong, W.; Cui, X.; Ma, J.; Li, S. Long non-coding RNA FGD 5-AS1 promotes nonsmall cell lung cancer cell proliferation through sponging hsa-miR-107 to up-regulate FGFRL1. Biosci. Rep. 2002, 40, BSR20193309. doi:10.1042/BSR20193309

27. Zhang, R.; Niu, Z.; Pei, H.; Peng, Z. Long noncoding RNA LINC00657 induced by SP1 contributes to the non-small cell lung cancer progression through targeting miR-26b-5p/COMMD8 axis. J. Cell. Physiol. 2020, 235, 3340-3349. doi:10.1002/jcp.29222

28. Lei, Y.; Wang, Y.H.; Wang, X.F.; Bai, J. LINC00657 promotes the development of colon cancer by activating PI3K/AKT pathway. Eur. Rev. Med. Pharmacol. Sci. 2018, 22, 6315-6323. doi:10.26355/eurrev_202103_25398

29. Yu, S.Y.; Peng, H.; Zhu, Q.; Wu, Y.X.; Wu, F.; Han, C.R.; Yan, B.; Li, Q.; Xiang, H.G. Silencing the long noncoding RNA NORAD inhibits gastric cancer cell proliferation and invasion by the RhoA/ROCK1 pathway. Eur. Rev. Med. Pharmacol. Sci. 2019, 23, 3760-3770. doi:10.26355/eurrev_201905_17802

\section{Supplementary Files}

This is a list of supplementary files associated with this preprint. Click to download.

- Cts.xlsx 